\title{
SUPERLUMINAL SOUND AND FERROMAGNETIC TRANSITION IN THE ZELDOVICH MODEL
}

\author{
G. KALMAN and S. T. LAI* \\ Dept. of Physics, Boston College, Chestnut Hill, Mass. 02167, U.S.A.
}

\begin{abstract}
The implications of the Zeldovich model (baryons interacting through a massive vector field) for the problem of superluminal sound propagation and ferromagnetic transition are examined. In a classical baryon gas at high densities correlation effects lead to the pressure increasing faster than the energy, ultimately resulting in superluminal sound; crystallization phase transition appears however at comparable densities, thus competing with the onset of superluminal sound. For a high density fermi gas the domains of ferromagnetic transition are delineated, indicating a minimal and maximal density below and above which no ferromagnetic transition can be expected. The latter is further affected by relativistic effects requiring a different approach to the calculation of exchange energy and of the ferromagnetic phase.
\end{abstract}

\section{Introduction}

The study of matter at ultra-high density poses some questions of fairly general character, the answers to which are expected to be virtually independent of the details of the interaction between the particles. Zeldovich's model has been devised (Zeldovich, 1962) and employed (Bludman and Ruderman, 1968) to investigate such questions. The model describes the interaction between nucleons with the aid of a massive vector field. The resulting repulsive short range potential is taken at its face value (without corrections resulting from the exchange of several mesons) and can be used both classically and quantum mechanically. It has been recently speculated (see the paper by Bethe in this volume) that it is indeed in the domain of very strong coupling where such a model provides a fairly reliable approach and a superposition of Zeldovich-like potentials has been used in several attempts (Leung and Wang in this volume) to describe realistically nuclear matter at ultrahigh $\left(\varrho>10^{15} \mathrm{~g} \mathrm{~cm}^{-3}\right)$ densities. Although our justification for using the Zeldovich model is not that we expect it to be a realistic nuclear potential, in order to fix ideas we might stipulate that we are dealing with densities $n>1 \mathrm{fm}^{-3}\left(\varrho>1.6 \times 10^{15} \mathrm{~g} \mathrm{~cm}^{-3}\right)$ with a vector meson of mass $\sim 770 \mathrm{MeV}$ (for $\omega$ meson $m_{\omega}=784 \mathrm{MeV}$ ) corresponding to a range of interaction $\mu^{-1}=0.26 \mathrm{fm}$ and a coupling constant $\mathrm{g}^{2} / \hbar c=10 \sim 20$.

In this paper we investigate the puzzle of superluminal sound propagation and the problem of ferromagnetic transition; we also comment on the question of high density crystallization. These points we studied in the context of the Zeldovich model: it is hoped that the investigation of the various aspects of a single model is illuminating, even though no immediate connection with concrete physical systems can be established.

\section{Superluminal Sound Propagation}

\subsection{EQuATION OF STATE}

As calculations for the equation of state for neutron matter at supernuclear and near-

* Now at Logicon Inc., False Church, Va. 22044, U.S.A. 
relativistic densities led to such high pressures that $p$ (pressure) became comparable to $\varepsilon$ (energy density, including rest mass), the question of sound velocity acquired some interest. To be sure, results indicating substantially superluminal sound velocities emerged (as in Barker et al., 1967, for example), but it was argued that they were due to nonrealistic hard core potentials, inconsistent use or complete neglect of relativity, etc., and it was generally held that in the ultrarelativistic limit $p \leqslant \varepsilon / 3$ should be obeyed, the equality being reached asymptotically. Correspondingly, since the speed of sound is determined by

$$
c_{\mathrm{S}}=c\left(\frac{\partial p}{\partial \varepsilon}\right)_{\mathrm{S}}^{1 / 2}
$$

it follows that the maximum sound speed would have been limited to $c_{\mathrm{S}} \leqslant c / \sqrt{ } 3$.

It has been demonstrated by Zeldovich (1962) that the consistent relativistic use of his model does not support this contention. Indeed, in the Hartree approximation which was used by Zeldovich both the maximum limiting $p / \varepsilon$ and $c_{\mathrm{S}} / c$ ratios approach one, viz.,

$$
\begin{aligned}
p / \varepsilon & \leqslant 1, \\
c_{\mathrm{S}} / c & \leqslant 1 .
\end{aligned}
$$

This result originates from the presence of the positive Hartree terms for a onecomponent system with repulsive short range interaction. The Hartree terms are of the order $0\left(n^{2}\right)$ as compared to $0(n)$ for the kinetic terms, and hence at extremely high densities the Hartree terms dominate, resulting in the asymptotic equation of state:

$$
\lim p \rightarrow \varepsilon .
$$

This leads to $\lim _{n \rightarrow \infty} c_{\mathrm{S}} \rightarrow c$, implying possible existence of luminal sound speed at extreme relativistic densities. Similar results on zero sound in a relativistic quantum gas described by the Zeldovich model were obtained by Kalman (1967).

Bludman and Ruderman (1968) went beyond the Hartree approximation by including correlations among particles in a crystal lattice. They showed that under these circumstances both ratios in (2) exceed unity and ultrabaric equation of state $(p>\varepsilon)$ and superluminal sound $\left(c_{\mathrm{s}}>c\right)$ obtained at sufficiently high densities.

It has been argued by Ruderman (1968) that the source of the acausal behavior which is at the root of the appearance of superluminal sound propagation - is connected with the inherent instability of a classical one-particle or many-particle system interacting with its own radiation field (cf. the problem of the classical runaway electron discussed in Rohrlich, 1965). In the detailed dynamical description of the system this indeed casts doubt on the validity of choosing and constructing Green functions in the customary way (Bludman and Ruderman, 1968; Ruderman, 1968); it is difficult to see, however, how such an argument will affect direct thermodynamical considerations. (Whether the conventional thermodynamic or statistical approach is valid when the retarded interaction between particles is dominant is, however, questionable. A study of the appropriate kinetic theory would be desirable.) 
Here we wish to report on our study of whether the introduction of correlations in the original Zeldovich model, where particles are in a gaseous (plasma) state, leads to superluminal rather than luminal sound propagation. The use of the word 'plasma' is indeed appropriate in the present context, since the behavior and the proper description of a system we are contemplating (a gas of 'charged' classical elementary particles at a finite temperature, interacting through a massive vector field) and that of

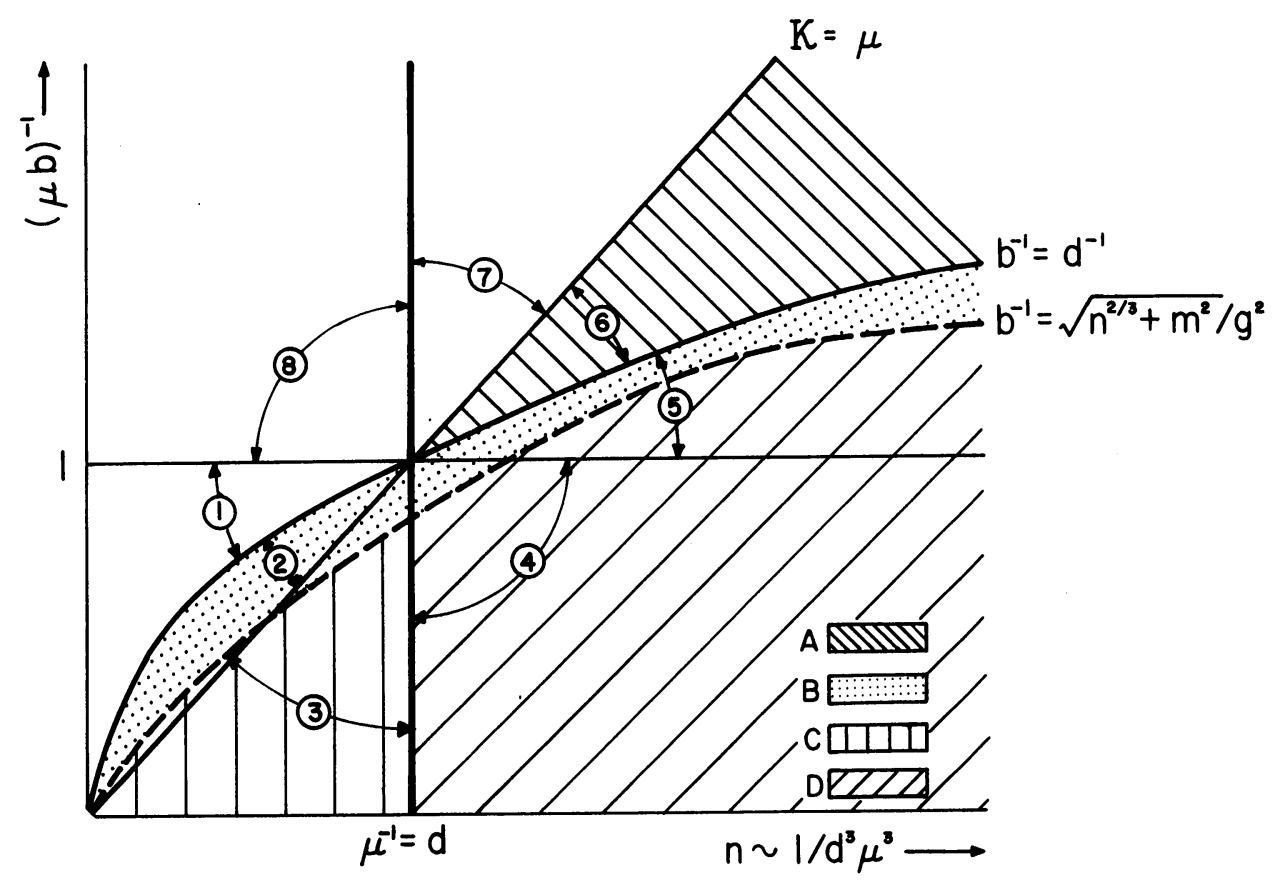

Fig. 1. Various domains in the parameter plane: for fixed values of the coupling constant and of the range of the field the parameter along the horizontal axis designates the density and the one along the vertical axis designates the temperature. The numbers enumerate the domains of different physical behaviors. Different shadings distinguish between the plasma domain (A), the classical lattice domain (B); the domain of fermi gas behavior (C), and the domain of quantum crystal behavior.

a classical electrodynamic plasma have much in common. To be sure, we are dealing with a system with a large coupling constant, but since we are studying the competitive roles, of Hartree and correlation effects, the plasma approach provides a good qualitative description of and a reliable physical insight into the fundamental behavior of the system.

Our answer to the question of whether superluminal sound appears in the present model is in the affirmative: we do get $c_{\mathbf{s}}>c$ when correlations become strong enough, although our method is not quantitatively reliable in the domain where this is obtained. We also acquire detailed descriptions of how correlation effects modify the sound speed, the equation of state and the stability of the plasma state. 
We now outline the essential ideas and steps of our derivation. The distribution function, $F(\mathbf{x}, \mathbf{p}, t)$, of a plasma in a 'self-consistent field approximation' is governed by the Vlasov equation:

$$
\left\{\frac{\partial}{\partial t}+\mathbf{v} \cdot \frac{\partial}{\partial \mathbf{x}}+g \mathbf{E} \cdot \frac{\partial}{\partial \mathbf{p}}\right\} F(\mathbf{x}, \mathbf{p}, t)=0,
$$

where $E_{\mu}$ is derived from the equations

$$
\begin{aligned}
& \left(\square-\mu^{2}\right) A_{\mu}=-4 \pi j_{\mu}, \\
& \mathbf{j}=g \int \mathbf{v} F(\mathbf{x}, \mathbf{p}, t) \mathrm{d} \mathbf{p}, \\
& \varrho=g \int F(\mathbf{x}, \mathbf{p}, t) \mathrm{d} \mathbf{p}, \\
& \mathbf{E}=-\dot{\mathbf{A}}
\end{aligned}
$$

From this system, the 'dielectric' response-function $\varepsilon$ of the system is obtained as:

$$
\begin{aligned}
\varepsilon(\mathbf{k}, \omega) & =1+\mathbf{k} \cdot \frac{\omega^{2}-k^{2}}{\omega^{2}-k^{2}-\mu^{2}} \frac{\omega_{0}^{2} m}{k^{2}} \int \mathrm{d} \mathbf{p} \frac{\partial F^{0} / \partial \mathbf{p}}{\omega-\mathbf{k} \cdot \mathbf{v}}, \\
\omega_{0}^{2} & =4 \pi \frac{g^{2} n}{m}
\end{aligned}
$$

where $F^{0}$ is the equilibrium distribution function. By invoking the fluctuationdissipation theorem (Kubo, 1957; Sitenko, 1967; Golden and Kalman, 1969), the pair-correlation function $G(\mathbf{k})$ becomes related to the imaginary part of the static response function $\varepsilon(\mathbf{k}, 0)$. We obtain (Kalman and Lai, 1972b)

$$
\begin{aligned}
G(\mathbf{k}) & =-\frac{\kappa^{4}}{4 \pi \beta} \frac{1}{k^{2}+\kappa^{2}+\mu^{2}}, \\
\kappa^{2} & =4 \pi g^{2} n \beta, \\
\beta & =1 / k T .
\end{aligned}
$$

The pair-correlation function leads to expressions for all the relevant thermodynamic quantities. Independently, the grand partition function is calculated by the cluster ring summation as in Brout and Carruthers (1963). This independent method verifies the thermodynamic results obtained earlier. The equation of state is given ultimately by

and

$$
\varepsilon(n)=\frac{1}{\beta}\left[3+\beta m G_{0}(\beta m)\right] n+\frac{1}{2}\left(\frac{4 \pi g^{2}}{\mu^{2}}\right) n^{2}-\frac{\kappa^{2}}{8 \pi \beta}\left[\left(\kappa^{2}+\mu^{2}\right)^{1 / 2}-\mu\right],
$$

$$
\begin{array}{r}
p(n)=\frac{n}{\beta}+\frac{1}{2}\left(\frac{4 \pi g^{2}}{\mu^{2}}\right) n^{2}-\frac{1}{3} \frac{\kappa^{2}}{8 \pi \beta}\left[\left(\kappa^{2}+\mu^{2}\right)^{1 / 2}-\mu\right]+ \\
-\frac{\mu}{24 \pi \beta}\left[\left(\kappa^{2}+\mu^{2}\right)^{1 / 2}-\mu\right]^{2}
\end{array}
$$


Expansions in density $n$ reveal ultrabasic behavior $(p>\varepsilon)$ at high densities. At infinite densities, the Zeldovich result is recovered.

To calculate sound speed $c_{\mathbf{S}}$, one can handle the partial derivatives by the Jacobian method (Landau and Lifshiftz, 1959), which leads to

$$
\left(\frac{\partial p}{\partial \varepsilon}\right)_{S}=\frac{\left[\left(\frac{\partial p}{\partial T}\right)_{V}^{2} /\left(\frac{\partial S}{\partial T}\right)_{V}-\left(\frac{\partial p}{\partial V}\right)_{T}\right]}{\frac{1}{V}\left(p+\frac{E}{V}\right)} .
$$

Alternatively, $(\partial p / \partial \varepsilon)_{S}$ can be calculated from the thermodynamic functions $p(n, \beta)$, $\varepsilon(n, \beta)$, and $S(n, \beta)$ by expressing $\beta$ in terms of $n$ at constant entropy $S$. Then, keeping $S$ constant, we have

$$
\left(\frac{\partial p}{\partial \varepsilon}\right)_{\mathrm{S}}=\left\{\frac{\mathrm{d} p[n, \beta(n, S)]}{\mathrm{d} n}\right\} /\left\{\frac{\mathrm{d} \varepsilon[n, \beta(n, S)]}{\mathrm{d} n}\right\} .
$$

At high densities, superluminal behavior emerges, because (10) or (11) yield

$$
\left(\frac{\partial p}{\partial \varepsilon}\right)_{\mathrm{S}}=1+\frac{2}{9} \mu^{2} g \beta^{1 / 2} \pi^{-1 / 2} n^{-1 / 2}+0\left(n^{-1}\right) .
$$

As $n \rightarrow \infty$, Zeldovich's result is again recovered, viz.,

$$
\lim _{n \rightarrow \infty}\left(\frac{\partial p}{\partial \varepsilon}\right)_{s} \rightarrow 1
$$

These results are qualitatively similar to those obtained by Bludman and Ruderman (1968).

As the density in the plasma state is increased beyond the plasma domain, symptoms of van der Waals type phase transition manifest themselves through the non-monotonic behavior of the pressure and energy density as functions of density. Whether the ensuing phase transition leads to a liquid or crystal structure cannot, of course, be determined at this point. Once the phase transition occurs the plasma approximation scheme evidently breaks down and no further prediction concerning the value of the sound velocity can be made. In the 'plasma domain' where our results are rigorously justified, neither ultrabaric equation of state nor superluminal sound result from (10), (11), and (12).

\subsection{HIGHER ORDER CORRELATIONS}

In order to somewhat refine our estimates, we consider the higher-order correlations by taking into account the 'watermelon' diagrams in the expansion of the partition function (Abe, 1959). These diagrams are of the order $\beta^{3 / 2} n^{1 / 2} g^{3}$. The higher-order 
correlations contribute to the free energy $F$ the correction term $F_{\mathrm{c}}$ given by

$$
F_{\mathrm{c}}=\frac{n^{2}}{2 \beta} \sum_{m=3}^{\infty} \int \mathrm{d} \mathbf{r}_{1} \mathrm{~d} \mathbf{r}_{2}\left\{-\beta g^{2} \frac{\exp \left[-\left(\kappa^{2}+\mu^{2}\right)^{1 / 2} r\right]}{r}\right\}^{m} \frac{1}{m !}
$$

Explicit evaluation of this infinite series of cluster integrals leads to corrections of pressure and energy density,

$$
\begin{aligned}
p_{\mathrm{c}} & =\frac{n}{\beta} \frac{\lambda^{2}}{12}\left(\log 3 \Lambda+\frac{\gamma}{2}-\frac{11}{6}+\frac{1}{2} \frac{\mu^{2}}{\kappa^{2}+\mu^{2}}\right) \\
\varepsilon_{\mathrm{c}} & =3 p_{\mathrm{c}}-\frac{\left(\kappa^{2} \mu g\right)^{2}}{48 \pi\left(\kappa^{2}+\mu^{2}\right)}, \\
\lambda & \equiv \kappa \beta g^{2} \quad \text { and } \Lambda \equiv\left(\kappa^{2}+\mu^{2}\right)^{1 / 2} \beta g^{2}, \quad \gamma=\text { Euler constant } .
\end{aligned}
$$

Since higher-order correlation terms are small refinements to the ring results, they do not cause any significant alteration in our previous finding concerning the physical behavior of the system.

The correction term to the sound speed is obtained as

$$
\Delta_{\mathrm{c}}\left(\frac{\mathrm{d} p}{\mathrm{~d} \varepsilon}\right)_{\mathrm{s}}=\frac{1}{4 \pi}\left[\frac{5}{6}\left(\frac{\log 3}{2}+\gamma-\frac{2}{3}\right)+\frac{13}{72}\right] \lambda \frac{\kappa^{3}}{n}+0\left(\lambda \frac{\kappa^{3}}{n} \log \lambda\right)
$$

where $\gamma$ is Euler's constant. This result shows that as long as $\left(\kappa \beta g^{2}\right) \kappa^{3} n^{-1}<1$, the correction is an insignificantly small negative term and the sound speed is overestimated in the ring approximation. There is no substantial modification of the phase transition criterion either.

\subsection{COMMENTS AND CONCLUSIONS}

As demonstrated in our model calculations, the emergence of ultrabaric behavior and superluminal sound stems from the fact that while correlations reduce both the pressure and energy density, the former is less affected thus increasing the value of $\mathrm{d} p / \mathrm{d} \varepsilon$. As the density and thus correlations are increased, phase-transition competes with the emergence of superluminal sound. Ultrabaric behavior occurs after superluminal sound, since at low densities energy density is always greater than pressure $p$, and hence the $p(n)$ curve has to climb at a steeper rate than $\varepsilon(n)$ in order to catch up with the latter.

It is instructive now to compare the densities where the superluminal and the ultrabaric behaviors occur with the critical density for the phase transition. For (9) and (11) we find that $p / \varepsilon=1$ for

$$
n_{\mathrm{ultra}}=\frac{9}{\pi} \frac{1}{g^{6} \beta^{3}}+\cdots
$$


and $\partial p / \partial \varepsilon=1$ for

$$
n_{\text {super }}=\frac{9}{\pi} \frac{1}{g^{6} \beta^{3}} .
$$

So far as the phase transition is concerned, a somewhat more detailed examination of (9) leads to

$$
n_{\mathrm{crit}}=\frac{1}{12 \sqrt{3 \pi}} \mu^{3},
$$

where $n_{\text {crit }}$ is the density at the critical point on the phase transition curve (Figure 2). The more detailed behavior of these quantities is illustrated in Figure 2. The general

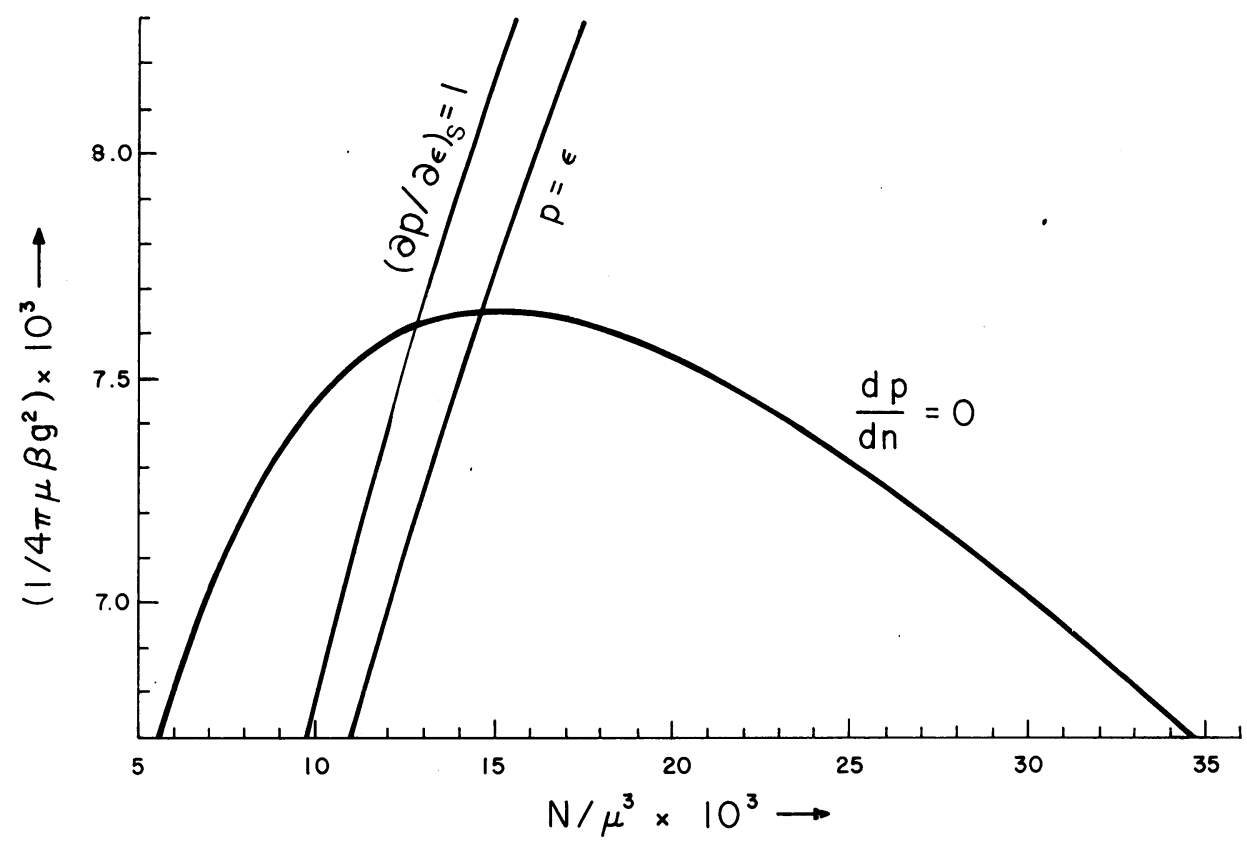

Fig. 2. Domains of phase transition ultrabaric and superluminal behavior. Note that they occur at comparable densities, but there are ultrabaric and superluminal domains outside the phase transition region at higher temperatures.

conclusion is that the ultrabaric and superluminal behaviors at high temperatures occur outside the phase transition domain, but in the region where short range correlations are important; thus the 'plasma' approximation is not really appropriate in this region. The qualitative conclusion, however, that the superluminal and ultrabaric behaviors do take place in the density and temperature domains indicated should not be affected by the more accurate description of correlations. 


\section{Ferromagnetic Transition}

\subsection{NONRELATIVISTIC}

The Zeldovich model provides an economic approach by which we can study the qualitative features of and the conditions for the ferromagnetic phase transition in a fairly realistic interaction model for nuclear matter at high densities. In particular, as the density approaches the relativistic region the spin-spin and transverse interaction (inherent in the vector character of the model) substantially modify the role of the exchange energy.

At non-relativistic densities the one-particle Hartree-Fock (HF) energy $\eta(p)$ is given by (Lai, 1970; Kalman and Lai, 1971)

$$
\eta(\mathbf{k})=\frac{h^{2} k^{2}}{2 m}+\frac{2 \pi g^{2}}{\mu^{2}}-\int_{\left|\mathbf{k}^{\prime}\right|<k_{\mathbf{F}}} \frac{\mathrm{d} \mathbf{k}^{\prime}}{(2 \pi)^{3}} \frac{4 \pi g^{2}}{\left|\mathbf{k}-\mathbf{k}^{\prime}\right|^{2}+\mu^{\prime 2}}
$$

The Hartree term is spin independent. The competition between the kinetic and exchange terms determines whether the system favors a ferromagnetic state. If the spin populations $\mathrm{N}_{ \pm}$are unequal, the system is ferromagnetic with magnetization $\zeta=\left(\mathrm{N}_{+}-\mathrm{N}_{-}\right) /\left(\mathrm{N}_{+}+\mathrm{N}_{-}\right)$. By comparing the average energy

$$
E(\zeta)=\sum_{\uparrow \downarrow} \frac{\mathrm{d} \mathbf{k}}{(2 \pi)^{3}} \eta(\mathbf{k} ; \zeta)
$$

per particle for $\zeta=0$ and $\zeta \neq 0$, the lowest energy state at a given density can be determined.

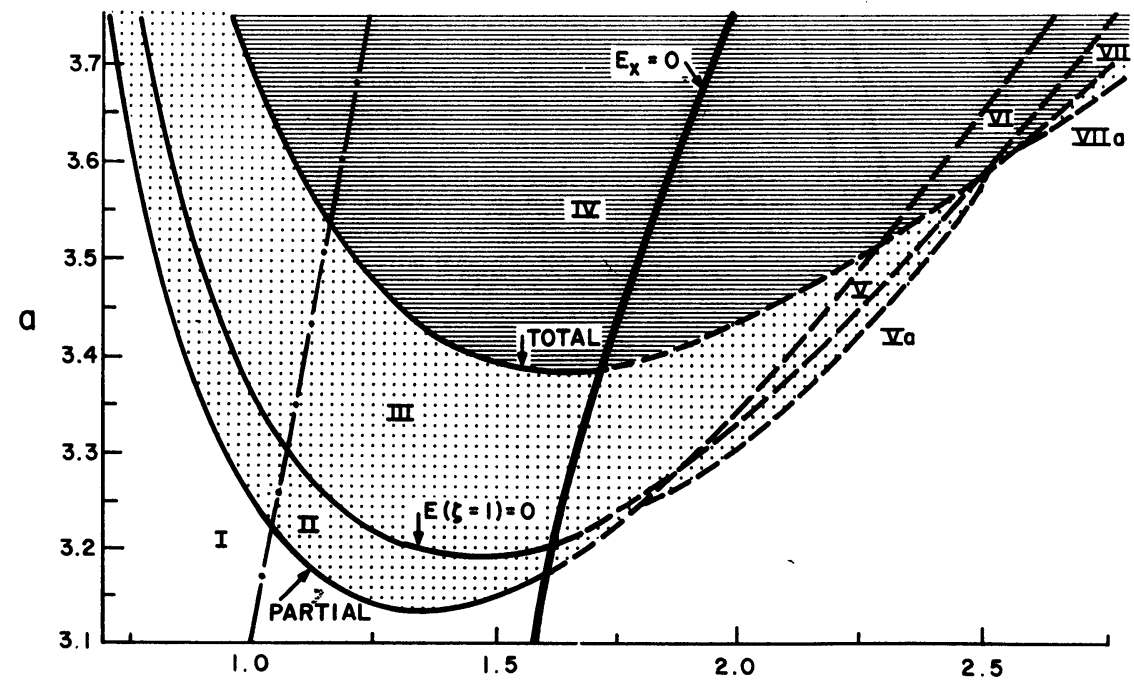

Fig. 3. Various boundaries of ferromagnetic transitions plotted against $a \equiv 2 m g^{2} / \mu \pi h^{2}$ and $x \equiv 2 k_{\mathrm{F}} / \mu$. The dash-dot line corresponds to the quantum crystallization density as estimated on the basis of the calculation by Canuto and Chitre (in this volume). The heavy solid line corresponds to $E_{x}=0$. 
Starting with low densities the lowest energy state is at $\zeta=0$ until the density is sufficiently high so that the location of $\min [E(\zeta)]$ moves away from $\zeta=0$. Thus the condition for the onset of partial magnetization is $\partial E /\left.\partial \zeta\right|_{\zeta=0}=0$ and $\partial^{2} E /\left.\partial \zeta^{2}\right|_{\zeta=0}=0$. Total magnetization is attained when $\min [E(\zeta)]$ is located at $\zeta=1$, i.e.,

$$
\partial E /\left.\partial \zeta\right|_{\zeta=1}=0 \text { and } \partial^{2} E /\left.\partial \zeta^{2}\right|_{\zeta=1}>0 \text {. }
$$

The results are shown in Figure 3. Ferromagnetism is favored at intermediate densities because in the low density regime where $\mu^{-1}$ is small compared to the interparticle distance $d$, the system behaves like one with $\delta$-function interaction while at high densities where $\mu^{-1}>d$ the system resembles a Coulomb gas.

Correlation induced phase transitions are obviously absent in the Hartree-Fock (HF) model. A HF-type phase transition (Gartenhaus and Stranahan, 1965a, b) is also absent in this case, since the presence of the Hartree terms maintains $\mathrm{d} p / \mathrm{d} n$ positive, both in the normal and ferromagnetic state.

\subsection{RELATIVISTIC FERROMAGNETISM}

At relativistic dersities $\left(\gtrsim 10^{15} \mathrm{~g} \mathrm{~cm}^{-3}\right)$ the problem of exchange energy in a fermi gas exhibits some unconventional features. The new physical aspects of the problem are the 'retardation,' i.e., the explicit energy dependence of the interaction potential and the importance of the transverse interaction; in addition, certain formal difficulties arise because of the description of the system in terms of helicity rather than spin states (Salpeter, 1961 ; Zapolsky, 1960).

As a result, the nonrelativistic exchange part of the HF Hamiltonian $H_{\mathbf{X}}^{\mathrm{NR}}$ is modified to $H_{\mathbf{X}}^{\mathbf{R}}$ as follows (Kalman and Lai, 1972a):

where

$$
\begin{aligned}
H_{\mathbf{X}}^{\mathrm{NR}} & =\left|\begin{array}{l}
\mathbf{p}_{1} S_{1} \\
\mathbf{p}_{2} S_{2}
\end{array}\right\rangle\left\langle\begin{array}{l}
\mathbf{p}_{1} S_{1} \\
\mathbf{p}_{2} S_{2}
\end{array}\left|\phi\left(\mathbf{p}_{1}, \mathbf{p}_{2}\right)\right| \begin{array}{l}
\mathbf{p}_{2} S_{2} \\
\mathbf{p}_{1} S_{1}
\end{array}\right\rangle\left\langle\begin{array}{l}
\mathbf{p}_{2} S_{2} \\
\mathbf{p}_{1} S_{1}
\end{array}\right|, \\
H_{\mathbf{X}}^{\mathbf{R}} & =\left|\begin{array}{l}
\mathbf{p}_{1} h_{1} \\
\mathbf{p}_{2} h_{2}
\end{array}\right\rangle\left\langle\begin{array}{l}
\mathbf{p}_{1} h_{1} \\
\mathbf{p}_{2} h_{2}
\end{array}\left|\phi^{\mathrm{R}}\left(\mathbf{p}_{1}, \mathbf{p}_{2}\right)\right| \begin{array}{l}
\mathbf{p}_{2} h_{2} \\
\mathbf{p}_{1} h_{1}
\end{array}\right\rangle\left\langle\begin{array}{l}
\mathbf{p}_{2} h_{2} \\
\mathbf{p}_{1} h_{1}
\end{array}\right|,
\end{aligned}
$$

$$
\begin{aligned}
\phi\left(\mathbf{p}_{1}, \mathbf{p}_{2}\right) & =\frac{g^{2}}{\left|\mathbf{p}_{1}-\mathbf{p}_{2}\right|^{2}+\mu^{2}} \\
\phi^{\mathrm{R}}\left(\mathbf{p}_{1}, \mathbf{p}_{2}\right) & =\frac{g^{2}\left(1-\alpha_{1} \cdot \boldsymbol{\alpha}_{2}\right)}{\left|\mathbf{p}_{1}-\mathbf{p}_{2}\right|^{2}-\left[\eta\left(\mathbf{p}_{1}\right)-\eta\left(\mathbf{p}_{2}\right)\right]^{2}+\mu^{2}}
\end{aligned}
$$

and $S$ stands for spin, $h$ for helicity.

The properties of the relativistic exchange integrals are displayed in Figure 4 . In the extreme relativistic region the exchange energy becomes positive in contrast to the negative value it has in the non-relativistic region.

In Figure 5 we have indicated the boundary where the exchange energy changes sign. No ferromagnetic behavior in the region to the right of the boundary can be expected and the likelihood for the ferromagnetic state corresponding to a lower energy diminishes as one approaches the boundary. The strong range-dependence of the 


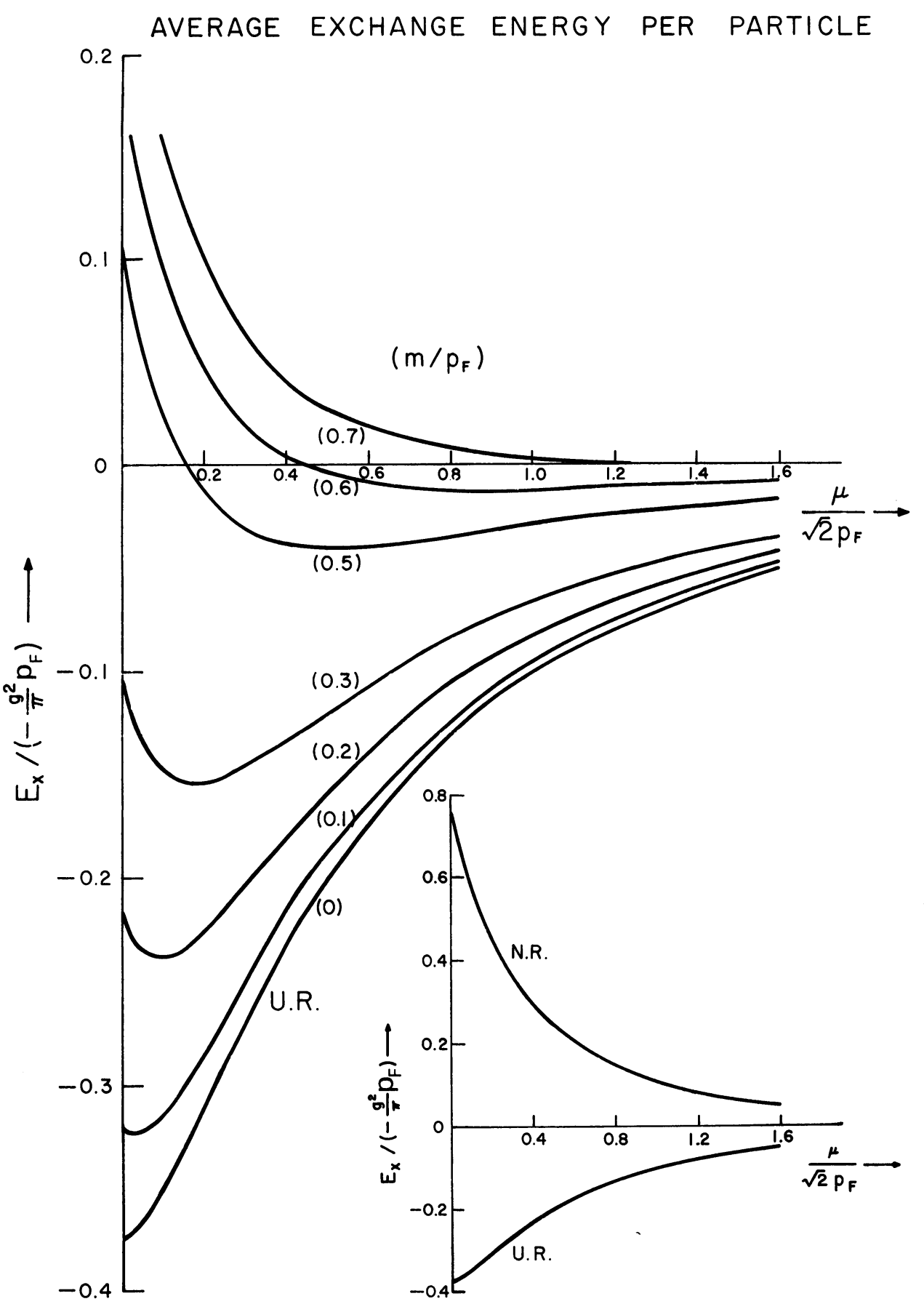

Fig. 4. Dependence of exchange energy $E_{x}$ on the range $\mu^{-1}$ of the field and the fermi momentum $p_{F}$. At sufficiently high densities, exchange energy becomes positive. The small insert depicts the transition boundary of $E_{x}$. 


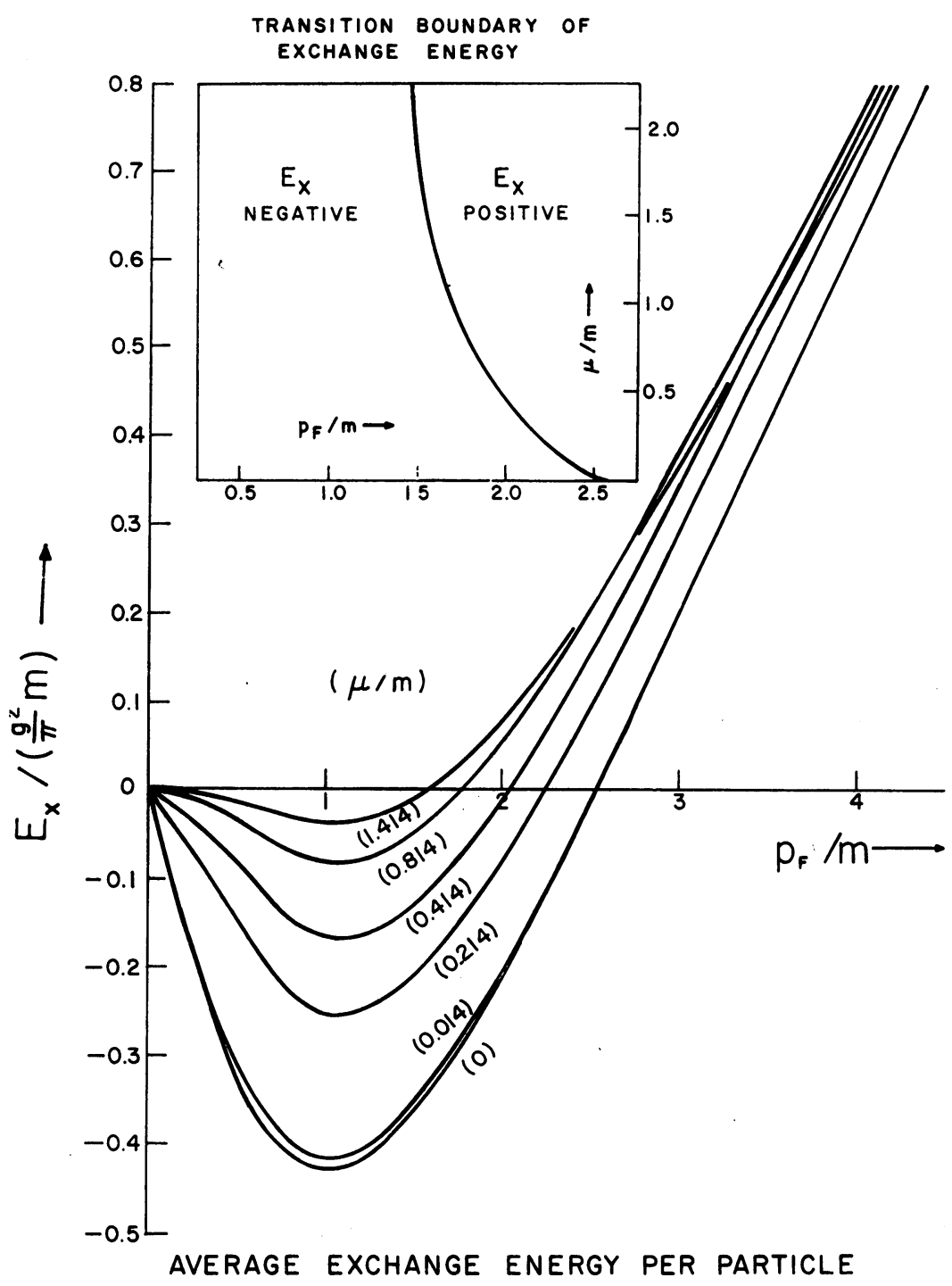

Fig. 5. Dependence of exchange energy on the range $\mu^{-1}$ of the field and the mass $m$ of baryon. The small insert illustrates the comparison of the non-relativistic and the ultrarelativistic behaviors.

density value where the transition occurs should be noted. For example, for an electron gas the exchange energy changes sign at about $15.6 n_{\mathrm{c}}\left(n_{\mathrm{c}}=m^{3} c^{3} / 3 \pi^{2} \hbar^{3}\right.$, the critical density for relativistic degeneracy) while for $\mu / m \simeq 0.8$ this takes place around $5.4 n_{\mathrm{c}}\left(n_{\mathrm{c}}=5.7 \times 10^{15} \mathrm{~g} \mathrm{~cm}^{-3}\right.$ for a neutron gas); however around $5 \times 10^{15} \mathrm{~g} \mathrm{~cm}^{-3}$ the relativistic decrease amounts to about $60 \%$ of the non-relativistically calculated value.

\subsection{Methodological QUeSTIONS}

In the relativistic domain the system is properly described in terms of helicity eigen- 
states: the ferromagnetic state which requires the spin rather than the helicities to take up a certain value is not an eigenstate of the HF Hamiltonian (within positive energy states). The proper definition of the ferromagnetic state now can be accomplished by considering a coherent superposition $|F\rangle$ of helicity states which maximizes the expectation value $\left\langle F\left|\Sigma_{3}\right| F\right\rangle$. Setting

one finds

$$
|F\rangle=\frac{1}{\left(1+b^{2}\right)^{1 / 2}}\{|p \uparrow\rangle+b|p \downarrow\rangle\}
$$

$$
b=-\frac{\varepsilon}{m} \cot \theta+\left(\frac{\varepsilon^{2}}{m^{2}} \cos ^{2} \theta+1\right)^{1 / 2}
$$

with $\varepsilon=\left(p^{2}+m^{2}\right)^{1 / 2}$ and $\theta$ being the angle between $\mathbf{p}$ and the $z$-axis. The maximum magnetic moment per particle now becomes

$$
\langle\mu\rangle_{\max }=\frac{e \hbar}{m c} \frac{m^{2}}{2 \varepsilon^{2}}\left(1+\frac{\varepsilon}{m}\right)\left(\frac{\varepsilon^{2}}{m^{2}} \cos ^{2} \theta+\sin ^{2} \theta\right)^{1 / 2} .
$$

A further problem arises if one wishes to apply the HF approximation in a consistent fashion, which requires that the one-particle Hamiltonian (including the exchange term) be diagonalized. This is automatically satisfied in the nonrelativistic approximation where the $|\mathbf{p} S\rangle$ base states diagonalize the exchange term [cf. (20)]. The latter however, is not diagonalized by the relativistic $|\mathrm{p} h r\rangle$ ( $r$ designates the signature of the energy) base states: therefore a transformation to a new set of states, say $|\mathbf{p} \chi \varrho\rangle$, is required,

$$
\underset{\text { exch }}{|\mathbf{p} \chi \varrho\rangle}=\sum C_{\chi \varrho, h r}|\mathbf{p} h r\rangle
$$

in such a manner that the expansion coefficients $C$ are to be determined self-consistently. This can be done by expressing the exchange Hamiltonian as

$$
\begin{aligned}
& H_{p h r, \bar{p} \bar{h} \bar{r}}^{\text {exch }}=\sum_{\mathbf{p}^{\prime}} \phi\left(\mathbf{p}, \mathbf{p}^{\prime}\right) \delta_{\mathbf{p} \overline{\mathbf{p}}} n_{p^{\prime}} X_{h r, \bar{h} \bar{r}}\left(\mathbf{p}, \mathbf{p}^{\prime}\right) \\
& X_{h r, \bar{h} \bar{r}}=\sum_{\substack{h^{\prime}{ }^{\prime} \\
h^{\prime \prime} h^{\prime \prime}}}\left\langle\begin{array}{c}
h r \\
h^{\prime} r^{\prime}
\end{array}\left|\left(1-\alpha_{1} \cdot \alpha_{2}\right)\right| \begin{array}{c}
h^{\prime \prime} r^{\prime \prime} \\
h r
\end{array}\right\rangle \Omega_{h^{\prime} r^{\prime}, h^{\prime \prime} r^{\prime \prime}}
\end{aligned}
$$

with the aid of the density matrix $\Omega$

$$
\Omega_{h^{\prime} r^{\prime}, h^{\prime \prime} r^{\prime \prime}}=\sum_{\chi Q} C_{\chi Q, h^{\prime} r^{\prime}}^{*} n_{\chi} n_{e} C_{\chi Q, h^{\prime \prime} e^{\prime \prime}}
$$

The diagonalization of $X$ provides the new base states $|\chi \varrho\rangle$ and thus the expansion coefficients $C$; since $X$ is already an implicit function of the $C$-s through (28) and (29) this procedure provides the self-consistency condition for the $C$-s. This work is in progress: at the time of the writing of this paper we have no definitive result to report on. 


\section{Remarks on the Question of Crystallization}

The question of crystallization of matter at very high densities in the context of Zeldovich model is worth some further exploration. Some order-of-magnitude estimates can easily be given. The temperature-density region for a classical plasma state is such that the kinetic energy exceeds the potential energy and the particles behave collectively despite the short range of the field; these lead to the condition: $\left(\beta g^{2}\right)^{-1}<d<\kappa^{-1}<\mu^{-1}$. As shown in Figure 1, this condition can be satisfied at very high densities provided that the temperature is sufficiently high. On the other hand, the conditions for the existence of a classical crystal are such that the kinetic energy is less than the potential energy and the thermal energy is greater than the fermi energy; these lead to the conditions: $\left(\beta g^{2}\right)^{-1}<n^{1 / 3}$ and $\left(\beta g^{2}\right)^{-1}>\left(n^{2 / 3}+m^{2}\right)^{1 / 2}\left(\hbar c / g^{2}\right)$. If the last inequality is reversed, the system becomes a quantum crystal. The appropriate temperature-density regions for these conditions are shown in Figure 1. The criterion for the existence of a quantum crystal can be given as

$$
\frac{\mu c}{\hbar}<n^{1 / 3}<\frac{g^{2} m}{\hbar^{2}},
$$

i.e., the system should be in the 'Coulomb' region, but the interparticle distance should not be smaller than the 'Bohr radius.' These conditions are not contradictory for strong coupling $\left(g^{2} / \hbar c>\mu / m\right)$ and will not be violated even for extreme relativistic regions.

As the system approaches the critical density for phase transition the strong correlations will diminish the role of the exchange energy and in the crystalline state the fermi gas approach is obviously invalid. For the purpose of orientation, we have depicted in Figure 3 the line

$$
n_{\text {critical }}=\left[3.2 \times 10^{-2} \times \frac{g^{2} m}{\hbar^{2}}\right]^{3},
$$

suggested by the results obtained for the actual solidification boundary by the recent computations of Canuto and Chitre (as given in this volume) with realistic nuclear potentials.

\section{References}

Abe, R.: 1959, Prog. Theor. Phys. Kyoto 22, 213.

Barker, B. M., Bhatia, M. S., and Szamosi, G.: 1967, Nuovo Cimento 52B, 355.

Bludman, S. A. and Ruderman, M. A.: 1968, Phys. Rev. 170, 1176.

Brout, R. and Carruthers, P.: 1963, Lectures on Many-Electron Problems, Interscience, New York.

Gartenhaus, S. and Stranahan, G.: 1965a, Phys. Rev. Letters 14, 341.

Gartenhaus, S. and Stranahan, G.: 1965b, Phys. Rev. Letters 14, 621.

Golden, K. J. and Kalman, G.: 1969, J. Stat. Phys. 1, 415.

Kalman, G.: 1967, Phys. Rev. 158, 144.

Kalman, G. and Lai, S. T.: 1971, Phys. Letters 34A, 75.

Kalman, G. and Lai, S. T.: 1972a, The Problem of Exchange Energy in a Relativistic Electron Gas, paper presented at the Spring Meeting of The American Physical Society, New England Section. 
Kalman, G. and Lai, S. T.: 1972b, Ann. Phys. (N.Y.) 73, 19.

Kubo, R.: 1957, J. Phys. Soc. Japan 12, 570.

Lai, S. T.: 1970, Brandeis University Ph.D. Thesis.

Landau, L. D. and Lifshitz, E. M.: 1959, Statistical Physics, Pergamon Press, New York.

Rohrlich, F.: 1965, Classical Charged Particles, Addison Wesley, Reading.

Ruderman, M. A.: 1968, Phys. Rev. 172, 1286.

Salpeter, E. E.: 1961, Astrophys. J. 134, 669.

Sitenko, A. G.: 1967, Electromagnetic Fluctuations in Plasmas, Academic Press, New York. Zapolsky, H.: 1960, Cornell University Report (unpublished).

Zeldovich, Ya. B.: 1962, Soviet Phys. JETP 14, 1143. 